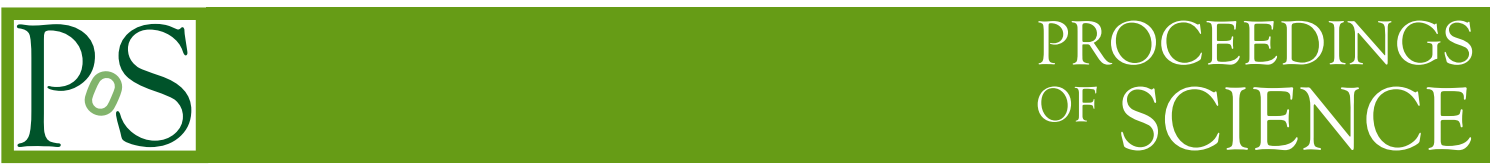

\title{
Cradle of Life
}

\section{Mario Tafalla*}

Observatorio Astronómico Nacional (Spain)

E-mail: m.tafalla@oan.es

\begin{abstract}
"Craddle of Life" is one of the key science drivers of the Square Kilometer Array, and explores the complex processes by which the conditions for life, and life itself, may become possible in the Universe. Of particular interest in this topic are the issues of planet formation via protostellar disk fragmentation and the search for extra-terrestrial intelligence.

On planet formation, the SKA, thanks to its very large sensitivity and resolution, will be the first instrument to spatially resolve the signatures of dust growth in the crucial $\mathrm{mm}$ and $\mathrm{cm}$ regime, providing important clues on the first stages in the build up of planetesimals. By working near its high-frequency end, the SKA is also expected to provide direct views of the process by which recently formed giant planets interact with the disk and open gaps of material via tidal forces. Time series of disk images may provide the first ever movies of planetary systems in formation, and should allow to follow the motion of disk asymmetries and tidal-induced spiral waves as the newly formed (and invisible) planets rotate around the star.

In the field of SETI, the SKA will bring a two orders of magnitude sensitivity improvement. It will not only increase the distance at which emitting civilizations could be detected, but will open for the first time the possibility of detecting unintentional emissions leaked by terrestrial analogs of TV signals and airport radars towards hundreds of stars in the solar vicinity.
\end{abstract}

2nd MCCT-SKADS Training School. Radio Astronomy: fundamentals and the new instruments August 26- September 4, 2008

Sigüenza, Spain

\footnotetext{
* Speaker.
} 


\section{Introduction}

Cradle of Life is one of the Key Science Projects of the Square Kilometer Array (SKA). It deals with old and fundamental questions like "Are we alone", or "Is life on Earth unique in the Universe?", which for centuries have lied closer to the realm of philosophy or religion than to the scientific enquiry. In the second half of the XX century, however, the identification of organic molecules in the interstellar medium (ISM), the first observations of protoplanetary disks around newly born stars, and the discovery of extrasolar planets around mature stars like our Sun, have brought the issue of life in the Universe to the realm of the questions addressable by observations, especially with new generation instruments. The SKA, thanks to its enormous sensitivity and resolution, promises to revolutionize our understanding of the possible existence of life outside Earth by providing us with exquisitely detailed images of planetary systems in the process of formation and by allowing us, for the first time, to extend the search for extra-terrestrial intelligence to technologies analog to ours. In this contribution I present a summarized version of these issues and the expected contribution of the SKA towards their solution. More detailed views of these topics can be found in the excellent reviews by [1], [2], and [3], on which these notes are based. These papers can be found, together with the full Scientific Case for the SKA, in the project web site www.skatelescope.org.

\section{From clouds to cores, from cores to stars with disks, and from disks to planets}

Although a number of gaps remain in our understanding of the complex process of star formation, a basic sequence of increasing density and central concentration is by now well established (see [4] for a detailed presentation of the state of the art in the field). The raw material for the process is the relatively diffuse gas that forms the so-called molecular clouds $\left(10^{2}-10^{3} \mathrm{~cm}^{-3}\right)$. These clouds present a variety of sizes and masses, and in all cases are characterized by highly irregular shapes and turbulent internal motions. By a still not well understood mechanism, the gas of the cloud condenses in some places forming pockets of higher density material $\left(\approx 10^{5} \mathrm{~cm}^{-3}\right)$ called dense cores (Fig. 1) which are believed to be the seeds in the process of star formation. The last decade or so has seen enormous progress in our understanding of the internal structure of these cores thanks to the combination of observations of their dust and gas components. These observations have revealed that the cores are centrally concentrated, but that the density distribution is relatively flat towards the center, and that in some cases, it agrees with the theoretical prediction for an isothermal sphere in the verge of gravitational equilibrium. The gas in the core evolves both in chemical composition (due to the freeze out of most molecular species onto the grains) and in its degree of concentration, towards the point of gravitational instability.

When instability occurs, the gas in the core starts to collapse gravitationally, leading to the formation of a protostellar object at the center. This object, invisible in optical light but already detectable through its millimeter and submillimeter emission, is in fact a complex system consisting of a hydrostatic core, a surrounding accretion disk, and a bipolar outflow that is ejected either from the disk surface or from the disk-star interface. While outflows can extend over a fraction of one parsec or so, and are therefore easily resolved with current instrumentation, disks are of the order of $100 \mathrm{AU}$ in size, so they span one arcsecond or less even in the most nearby star 

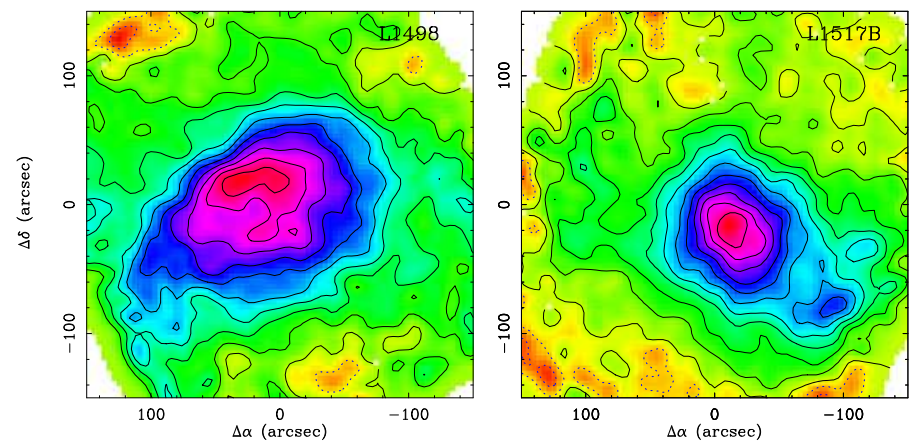

Figure 1: L1498 and L1517B, two starless cores in the Taurus-Auriga molecular cloud as observed in the $1.2 \mathrm{~mm}$ dust continuum emission. Note the central concentration that precedes the formation of a solar-type protostar.

forming regions like Taurus (140 pc away). Resolving the internal structure of disks is therefore a challenge to current instrumentation, and can only be done currently by direct observations at optical wavelengths in special occasions and through interferometric techniques in the infrared and radio regimes. The Hubble Space Telescope images of disks seen in silhouette against the bright Orion nebula, shown in Fig. 2, illustrate the power of high angular resolution observations, and confirm that disk formation is a necessary step in the sequence of star formation.

Accretion disks are expected to fragment and to form planets as they evolve. In our Solar System, the gaseous giant planets were formed several AU from the Sun, and even in extrasolar systems where we find large planets much closer to their stars (the so-called hot Jupiters), we believe that giant planet formation occurred much further away (5-10 AU from the stars), and that it was followed by inward migration due to planet-disk interaction. These AU scales of giant planet formation are unobservable with current instrumentation, but should be reachable by the SKA thanks to its enormous sensitivity and resolution.

\section{Contribution of the SKA to planet formation studies}

Protoplanetary disks are mostly gaseous, but the main player in the process of planet formation seems to be the dust, a component that initially contains about $1 \%$ of the mass and consists of submicron solid particles. The first step in the sequence of planet formation is the coagulation of these dust grains into millimeter and centimeter particles, followed by the buildup of larger and larger aggregates up to planetesimal (km-sized) objects.

Although directly unobservable, the first steps of the planet formation process can be inferred from studies of the dust emissivity, which is very sensitive to the size of the typical emitting particle. Here, the centimeter wavelengths reachable at the high-frequency end of the SKA range are of special interest. At these wavelengths, the dust emission should be optically thin, which is not the case at optical, infrared, and possibly millimeter wavelengths. SKA observations, therefore, will be sensitive to the full content of the disk, and not limited to the outer layers that are probed by other optically thick tracers. Centimeter-wavelength observations, in addition, present a special advantage in being sensitive to a critical regime of dust particles. Dust continuum observations at 


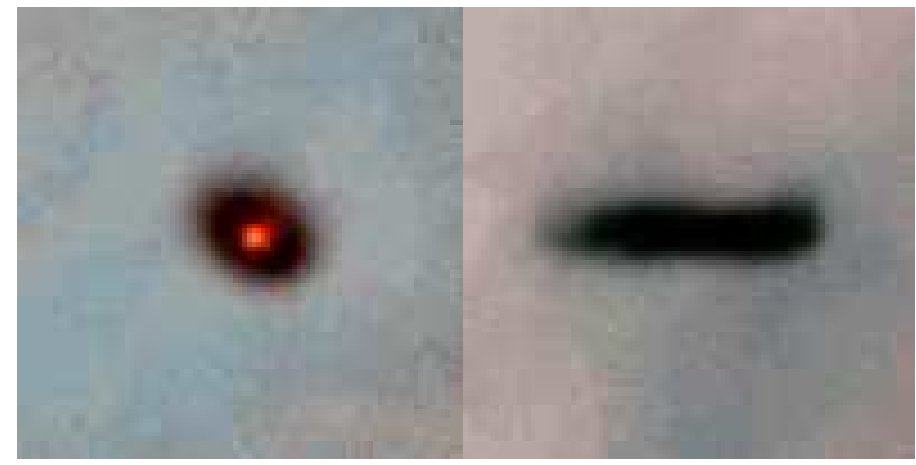

Figure 2: Two protoplanetary disks in the Orion cluster viewed in silhouette against the bright background provided by the ionized nebula. Hubble Space Telescope images courtesy Mark McCaughrean (MaxPlanck-Institute for Astronomy), C. Robert O’Dell (Rice University) y NASA.

a given wavelength are mostly sensitive to particles with similar size, so short cm wavelength SKA observations will be sensitive to the first stages of the dust coagulation process. These first stages in the $\mathrm{mm}$ and $\mathrm{cm}$ range are the most problematic in our understanding, as laboratory measurements suggest that large velocity collisions $\left(v>1 \mathrm{~m} \mathrm{~s}^{-1}\right)$ tend to destroy the grains, while slower collisions do not lead to sticking. Grain growth, however, does occur, and $\mathrm{mm}$ and $\mathrm{cm}$-sized particles are often inferred from disk spectral energy distributions. By mapping protoplanetary disks at different wavelengths in the $\mathrm{cm}$ range, the SKA will be able to tackle the grain-growth problem and characterize the spatial distribution of the grain population in disks.

Another issue in disk evolution where the SKA contribution will be significant is the study of the interaction between the disk and the newly-formed giant planets. Analytic calculations and computer simulations show that the tidal interaction between a massive planet and a disk leads to the opening of a gap. Disk material orbiting at radii larger than the planet gains angular momentum and moves outwards, while material orbiting interior to the planet losses angular momentum and spirals inward. As a result, a Jupiter-sized planet orbiting at $5 \mathrm{AU}$ from a Sun-like star is expected to open a disk gap having a width of close to $1 \mathrm{AU}$. The SKA operating at $22 \mathrm{GHz}$ of frequency and with baselines of up to $1500 \mathrm{~km}$ should achieve an angular resolution of about 2 milliarcsecs, or about $0.25 \mathrm{AU}$ at the distance of Taurus, and this should be enough to detect gaps. The SKA, therefore, could obtain the first unambiguous (albeit indirect) detection of the formation of giant planets in the nearest star-forming regions.

As the orbital motions of giant planets at a few AU from a Sun-like star have periods of a few years, multi-epoch SKA observations of protoplanetary disks separated by just few months should detect changes in the gap geometry, as the (invisible) planet orbits around the star. Time sequences ("movies") of the changes in disk asymmetries and planet-induced spiral waves should allow us not just to follow the motion of newly-formed planets in almost real time, but to test models of disk evolution and planet-disk interaction using both spatial and time-resolved observations. Current studies of extrasolar planets show an enormous variety of planetary systems in terms of semi-major axis, eccentricity, and the type of orbiting objects. The high frequency of hot Jupiters orbiting nearby stars, for example, suggests that our Solar System may belong to a rather restricted family 


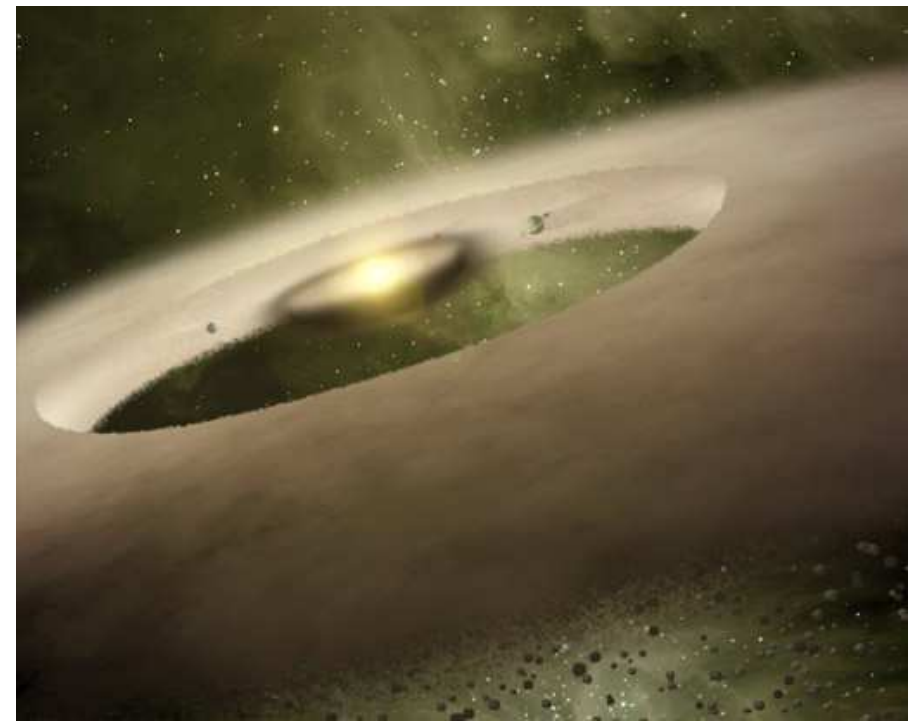

Figure 3: Artist view of the gap opened by planet formation in the disk around UX Tau A. Similar gaps could be directly imaged by SKA observations at the high frequency end of its range of operation. Image credit: NASA/JPL-Caltech.

of systems, and that strong disk-planet interaction may be more frequent than previously thought. SKA observations of a representative sample of protoplanetary disk systems may provide the first resolved images of these systems at their critical time of formation, and allow us to understand under what conditions a planetary system produces hot Jupiters or becomes similar to our Solar System.

\section{Contribution of the SKA to the Search for Extra-Terrestrial Intelligence}

As of the time of this lecture, we know of more than 300 extrasolar planets, and the number keeps growing almost weekly. Excluding the planetary system around the pulsar B1257+12, all other planets orbit "normal" stars, many of them like our Sun, with a prevalence of the so-called hot Jupiters due to a strong observational bias. These large giant planets orbiting at radii smaller than that of Mercury are favored by radial-velocity and transit searches, and therefore dominate the detection statistics in current surveys. Although it is not possible to detect Earth-type planets with present technology, indirect arguments suggest that these planets may be dominant in extrasolar planetary systems, and that a large fraction (one third to one half) of the stars in the Milky Way may in fact harbor terrestrial planets.

Existence of a terrestrial planet is one of the requirements for the development of life. Another one is the presence of liquid water, which sets a constraint in the orbital distance between the planet and its host star, and defines the so-called habitable zone. In our Solar System, the Earth is the only planet located in this special band. While we lack of statistics on how frequently this band is populated in other planetary systems, future observations by the Kepler space mission may soon provide the information on this critical question, and identify the first extrasolar planets with possible liquid water on their surface. In principle, we can expect a non-negligible fraction of all 
planetary systems having some object in the habitable zone, and therefore hosting an environment that we can consider friendly to life.

Whether a terrestrial planet with liquid water develops life, and on what time scale, is still a mystery, but there are hints that the process should not be unique to our planet. Life on Earth makes use of the most abundant elements in the Universe, mostly $\mathrm{H}, \mathrm{C}, \mathrm{O}$, and $\mathrm{N}$, and radio observations of the ISM show routinely widespread carbon chemistry in the molecular clouds of the Milky Way and other galaxies. In our Solar System, there is even evidence for amino acids produced in meteorites and comets. All this suggests that the chemistry of life can be repeated under a variety of forms and environments, and that there is no reason for life on Earth being unique in the Universe or in our galaxy.

Even if the Milky Way were teeming with life, detecting it outside our Solar System would be a formidable task. Indirect signatures like the presence of $\mathrm{O}_{3}$ (ozone) or $\mathrm{CH}_{4}$ (methane) in the atmosphere of terrestrial planets are still outside our technological reach, and their interpretation depends on our conviction that they may not originate from non biological processes. The Search for Extra-Terrestrial Intelligence (SETI) provides a very different approach by narrowing the search for life to only technologically advanced life. In this search, the radio regime plays a fundamental role, as it provides a very favorable channel for communications across the whole galaxy without the problem of extinction that affects optical and even IR wavelengths. Although there is no one a priori natural wavelength for SETI communication, considerations of minimal noise due to the galactic or microwave backgrounds suggests a frequency band between 1 and $10 \mathrm{GHz}$, which lies comfortably inside the reachable range of the SKA.

Thanks to its enormous collecting area and signal-processing capabilities, the SKA will revolutionize SETI work. More than 100 SETI searches have been carried out since Frank Drake's OZMA project in 1960, but sensitivity constraints have so far limited the search to technological civilizations that emit on purpose with the goal of communicating. This particular club of beaming civilizations (to which we do not belong) is clearly very limited, and its non detection only provides a very weak constraint on the existence of intelligent life in our galaxy. A more desirable approach would be to search for signals that leak into space unintentionally by equivalents of our radio and TV signals and airport radars, which are more likely to be emitted (more or less isotropically) by any civilization with our level of technology. This type of emissions have been out of reach from previous searches, but the two-orders of magnitude sensitivity improvement provided by the SKA will allow us to explore this new niche in several hundreds of nearby stars. Thus, in addition of allowing us to search towards literally billions of stars for civilizations emitting Arecibo-type beamed transmissions, the SKA will open the door to search for terrestrial analogs in the vicinity of the Solar System.

\section{References}

[1] Lazio, T. J. W., Tarter, J. C., \& Wilner, D. J. 2004, New Astronomy Review, 48, 985

[2] Tarter, J. C. 2004, New Astronomy Review, 48, 1543

[3] Wilner, D. J. 2004, New Astronomy Review, 48, 1363

[4] Reipurth, B., Jewitt, D., \& Keil, K. 2007, Protostars and Planets V, (Tucson: University of Arizona Press) 